PROBLEMS OF ENGINEERING CYBERNETICS AND ROBOTICS • $2021 \bullet$ Vol. 76, pp. 25-38

p-ISSN: 2738-7356; e-ISSN: 2738-7364

https://doi.org/10.7546/PECR.76.21.02

\title{
Designing of Technical Tools for Distributed Systems for Wireless Gathering, Transferring and Management of Information
}

\author{
Krasimir Markov \\ Institute of Information and Communication Technologies \\ at the Bulgarian Academy of Sciences \\ Acad. Georgi Bonchev Str., bl. 2, 1113 Sofia, Bulgaria \\ E-mail: krasikasi@abv.bg
}

\begin{abstract}
This paper presents the needed data for building a mathematical model of an electrical analogue filter. The described model is implemented in a MATLAB environment. Several different stages are discussed when constructing the electrical circuit: electrical circuit analysis, frequency analysis, time analysis, modulation of signals, and filtering of signals.
\end{abstract}

Keywords: Radio-frequency identification (RFID), Electromagnetic compatibility (EMC), Magnitude-phase representation (MPH), Electromagnetic susceptibility (EMS).

\section{Introduction}

The contemporary development of information and communication technologies resulted in the wide usage both of office and at the home [1]. Along with this, the GPS and Wi-Fi connectivity are part of the digital transformation related with Internet of Things [2]. A detailed review show that variety of technologies are needed to achieve smart farming by combining of image processing, machine learning, big data, cloud computing, and all of these are rely on the information from wireless sensor networks [3]. It is worth to mention that correct work of such devices should be secured by suitable model for cyber network security [4] including activities to support process migration in grid [5]. Some recent investigations points about the problems of Human Secure Interaction with the 
Internet Space [6], while other show the influence of electromagnetic fields on human brain [7]. That is why the electromagnetic signals and more precisely the electromagnetic compatibility (EMC) is a key factor for assure the proper devices work. EMC represents the capability of a certain electronic device to function satisfactory in its own electromagnetic environment, without creating interference on other devices in the same environment. The technical methods for suppressing, reducing and completely removing such interference and noise that it may create are the following: filtering, screening and grounding. These approaches are used in many signal processing and signal detection tasks. In papers [8-13] are used frequency analysis, time analysis and signal modulation for detection and parameter estimation of moving target and smart technologies for information sources support.

When electronic devices receive strong electromagnetic waves, unwanted electric currents can be induced in the circuit and thus cause unintended operations or interference with the intended operations. If the energy applied from the outside is too powerful, electronic devices can be damaged. Even if the energy applied from the outside is small, if it is mixed with the radio waves used for broadcasting and communication, it can cause loss of reception, abnormal noise, or disrupted at places where the radio waves for broadcasting and communication are weak. Such interference caused by external electromagnetic waves is called electromagnetic noise interference, and the electromagnetic waves that cause interference are called electromagnetic noise.

Noise can cause interference with various electronic devices. The source of noise also varies. Noise that does not cause any interference with particular appliances can seriously affect other devices. Therefore, there are rules to suppress the noise generated from electronic devices to a certain level and to make sure that electronic devices operate correctly under a certain noise level so that we can use electronic devices with security. These rules are called noise regulations.

If an electronic device is considered a source of noise, the occurrence of noise is called emission. In contrast, if an electronic device is considered a victim of noise, the tolerance to noise is called Electromagnetic susceptibility (EMS) [14].

The object of this paper are the technical methods for suppressing noise through filtering. For this purpose, the theoretical data of the human knowledge in the sphere are considered: signals and systems, frequency analysis, time analysis, signal modulation and signal filtering. Using the programming in MATLAB environment, the synthesis of all the different stages of the simulation process is shown. 


\section{Defining signals in MATLAB environment}

\subsection{Periodic signals}

The periodic signals are the signals that repeat after a certain time period, called repetition periods. The simplest example of that is called the sinusoidal (harmonic) oscillation which is analytically described with the famous equation $[15,16]$ :

$$
U=U_{m} \sin (w . t+\phi)
$$

Here $u$ is the moment value of the voltage, $U_{m}$ is the amplitude value of it, $\omega=2 \pi f$, is the circular frequency, $t$ is the time and $f$ is the initial phase. The period $T$ of repetition of the oscillation is connected to its frequency $f$ with this formula:

$$
T=\frac{1}{f}
$$

- Description of a periodical sinusoidal signal in the environment of MATLAB

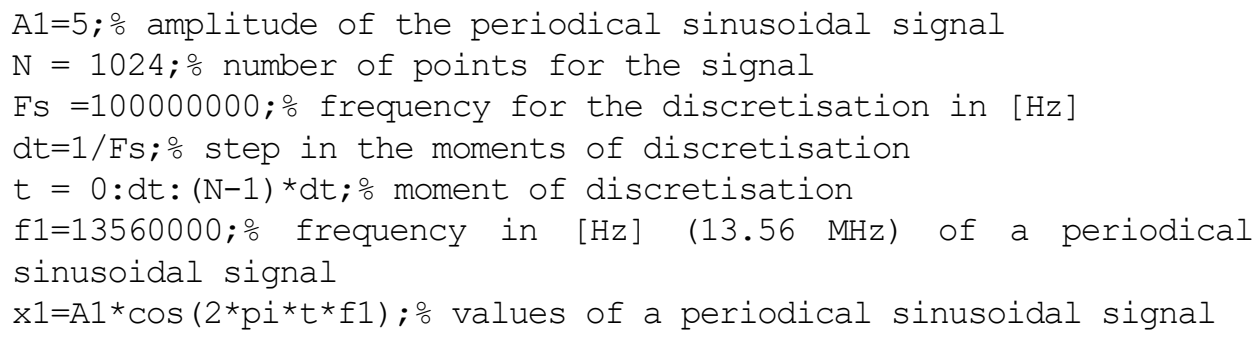

- Description of a periodical rectangular signal in the environment of MATLAB

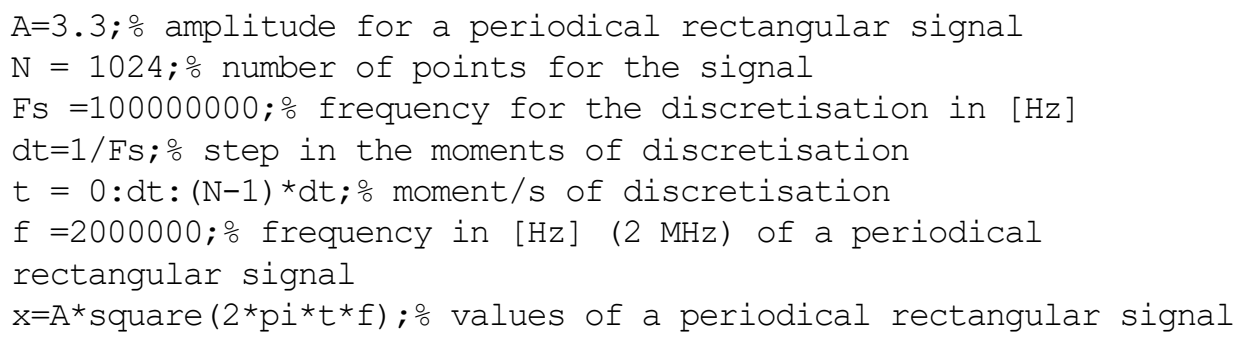

\subsection{Modulating periodical sinusoidal signals}

Modulation is a process of changing the parameters of a signal, called the carrying signal, under the influence of another one, called the modulating signal $[17,14]$. The carrying signal has a much higher frequency than the modulating one and is usually changed by the sinusoidal or cosine theorem. Depending on the parameter that is being modulated we can distinguish amplitude modulation (AM), 
frequency modulation (FM) and phase modulation (PM). The last two are more well-known with their joint name angular modulation. When the carrier signal has rectangular form, an impulse modulation occurs. As with the other type, this can also be split into amplitude impulse modulation (AIM), frequency impulse modulation (FIM), phase impulse modulation (PIM) and wide impulse modulation (WIM). When the carrier signal is harmonic, and the modulating one has a rectangular form, the terms of manipulation are similar - amplitude, frequency and phase.

The mathematical model of an amplitude modulated signal follows:

$$
A_{\text {ax }}(t)=A \cdot\left[1+m \cdot \cos \left(2 . \pi \cdot f_{0} \cdot t+\Psi_{0}\right)\right] \cdot \cos (2 \cdot \pi \cdot f \cdot t+\Psi)
$$

where $a(t)$ is carrying signal:

$$
a(t)=A \cdot \cos (2 . \pi \cdot f \cdot t+\Psi)
$$

where $\mathrm{a}_{0}(t)$ is modulating signal:

$$
a_{0}(t)=A \cdot \cos 0\left(2 . \pi \cdot f_{0} \cdot t+\Psi_{0}\right)
$$

The frequency of the modulating signal is much smaller than the one of the carrying signal $\left(\omega_{0} \ll \omega\right)$.

The following relation represents the modulation coefficient and represents a measure of the depth of a modulation:

$$
m=\frac{A_{0}}{A}
$$

For a successful transfer of the message sent, it (the coefficient) has to be less than $1(m<1)$. If $m>1$ the so called over modulation happens and on restoration of the initial signal at the receiver site, warping of the signal occurs. This leads to loss of information.

- Periodical amplitude modulated signal in the environment of MATLAB

The modulated signal is:

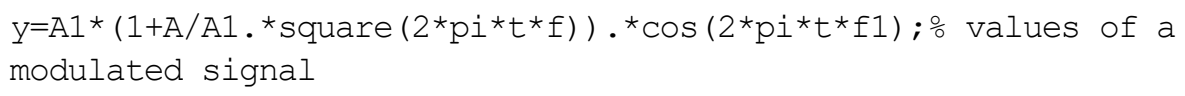

- Periodical amplitude modulated signal summed with a periodical sinusoidal periodical signal in the environment of MATLAB

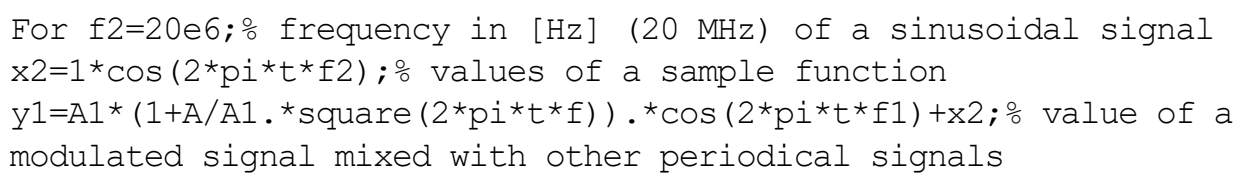




\section{Time and Frequency characterization of Periodic Signals}

\subsection{Fourier transform}

A random periodical signal $X(t)$ with a period of $T$ that meets the requirements of Dirichlet, [20,14], can then be described as a sum linear combination of complex exponents from a Fourier series:

$$
X(t)=a_{0}+2 \cdot \sum_{k=1}^{\infty} A_{K} \cdot \cos \left(k \cdot w_{0} t+\theta_{k}\right)
$$

From the two equations, it can be clearly seen that every periodical signal that meets certain requirements, can be described as a sum of constant part:

$$
a_{0}=\frac{1}{\mathrm{~T}} \cdot \int_{\mathrm{T}} X(t) \cdot d t
$$

and infinitely many harmonic parts:

$$
a_{k}=\frac{1}{\mathrm{~T}} \int_{T} x(t) e^{-j k w t} d t
$$

Every harmonic part in the Fourier series can be characterized with an amplitude $\mathrm{A}_{k}$ and initial phase $\theta_{k}$. The amplitude-frequency diagram is a graph of the frequency dependencies of the coefficients $A_{k}$ from the Fourier series.

The relation between the form (the time function) and the specter (the frequency function) of the signal is unambiguously and reversibly defined using the linear Fourier transform (LFT) and the inverse Fourier transform (IFT) in both its main forms - continuous and discrete.

\subsection{Graphical representation of signals as a time and frequency domain in MATLAB}

In this section it is shown in details the representation of signals in MATLAB environment for both time and frequency domain.

- Periodic sinusoidal signal described in item 2.1

\section{Time domain in MATLAB}

figure (1);

plot $(t, x 1) \circ$ graph of the time domain of the signal ' $x 1$ '

The time domain of the periodic sinusoidal signal described in item 2.1 is shown on Fig. 1. 


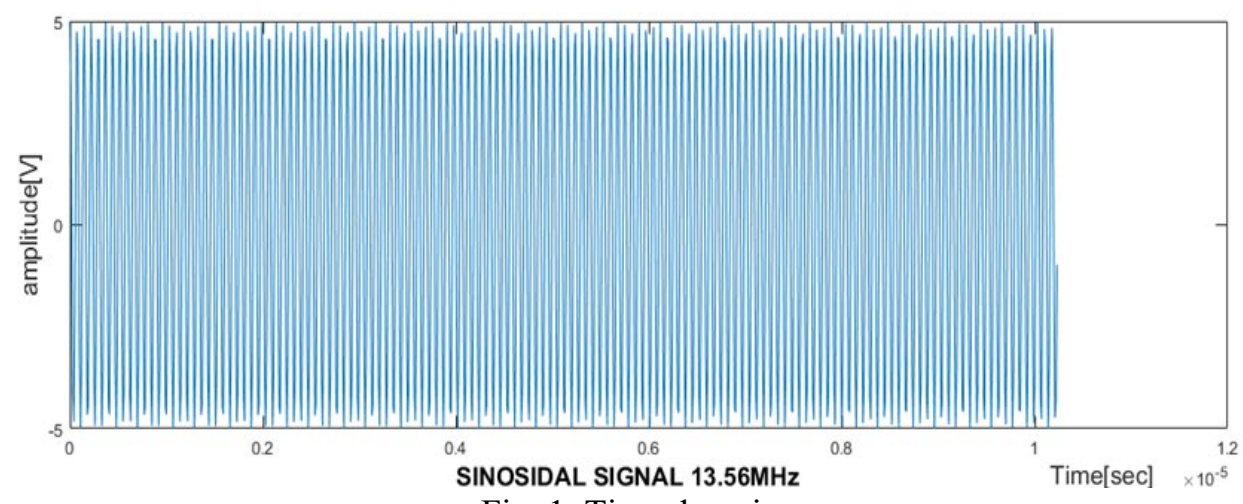

Fig. 1. Time domain

\section{Frequency domain in MATLAB}

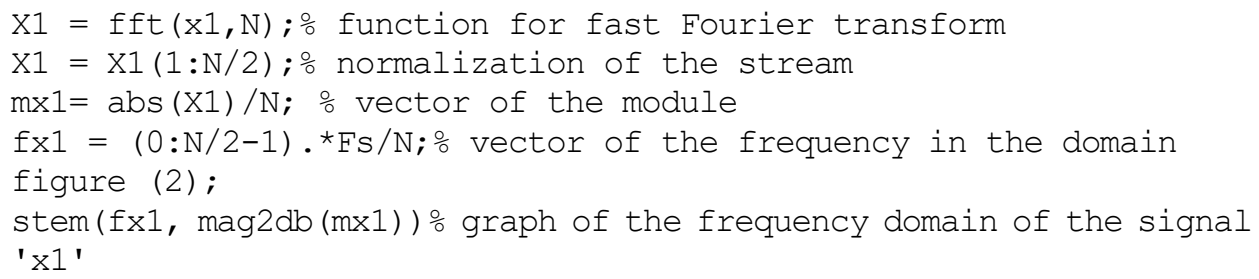

The frequency domain of the periodic sinusoidal signal described in item 2.1 is shown on Fig. 2.

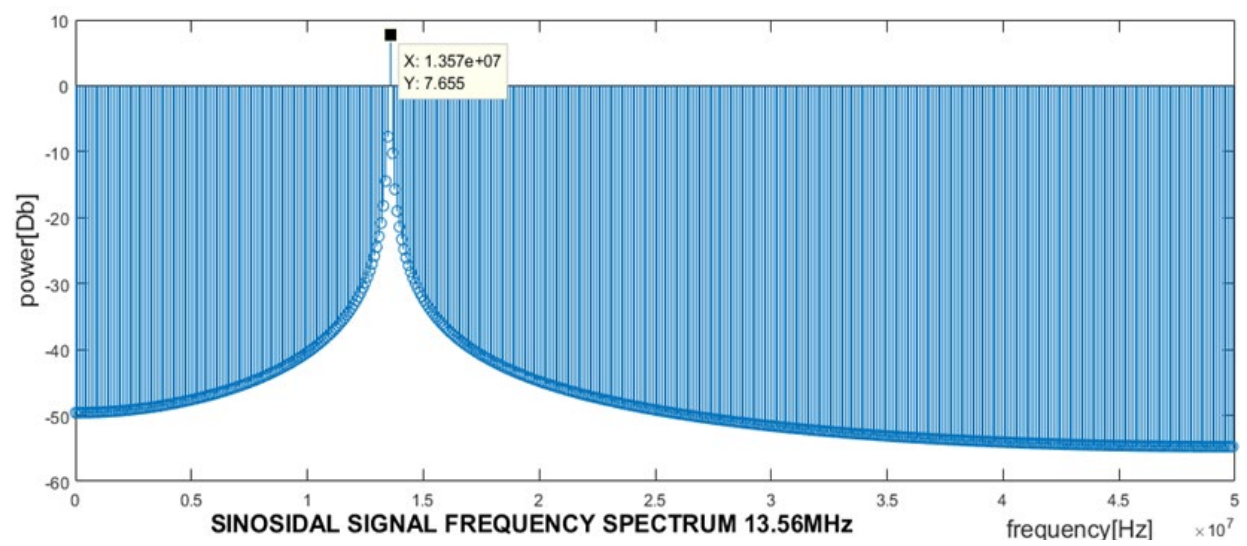

Fig. 2. Frequency domain

- Periodical rectangular signal described in item 2.1

\section{Time domain in MATLAB}

figure (3); $\%$

plot $(t, x) \circ$ graph of the time domain of the signal ' $x$ ' 
The time domain of the periodic rectangular signal described in item 2.1 is shown on Fig. 3.

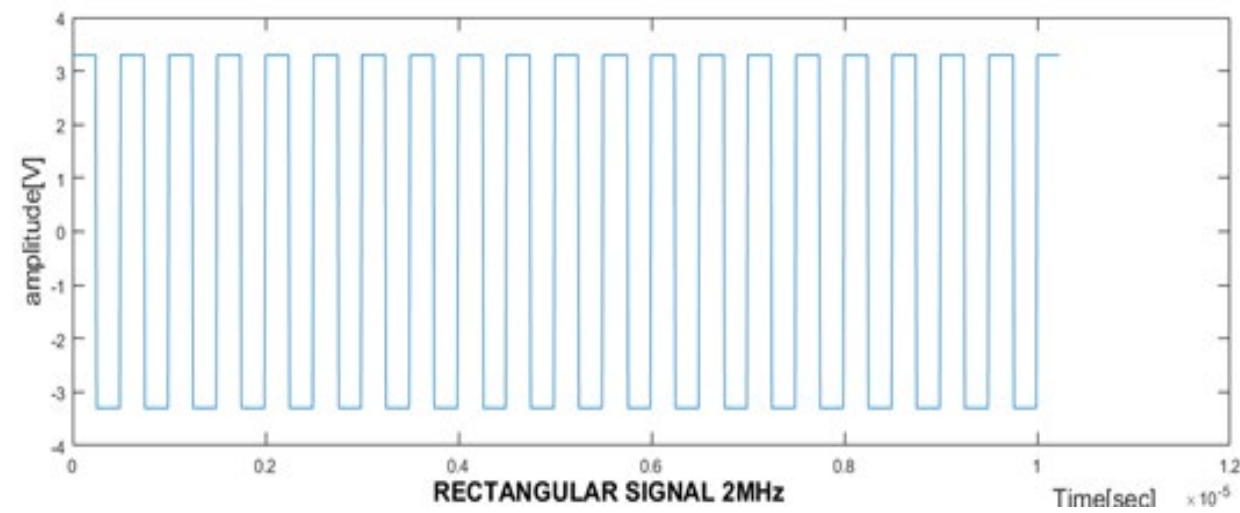

Fig. 3. Time domain

\section{Frequency domain in MATLAB}

$\mathrm{X}=\mathrm{fft}(\mathrm{x}, \mathrm{N}) ; \circ$ function for fast Fourier transform

$\mathrm{X}=\mathrm{X}(1: \mathrm{N} / 2)$; $\%$ normalization of the stream

$\mathrm{mx}=\operatorname{abs}(\mathrm{X}) / \mathrm{N}$; $\%$ vector of the module

$\mathrm{fx}=(0: \mathrm{N} / 2-1) \cdot{ }^{\star} \mathrm{Fs} / \mathrm{N}$; $\%$ vector of the frequency in the domain

figure (4);

stem( $f x, \operatorname{mag} 2 d b(m x)) \%$ graph of the frequency domain of the signal ' $x$ '

The frequency domain of the periodic rectangular signal described in item 2.1 is shown on Fig. 4.

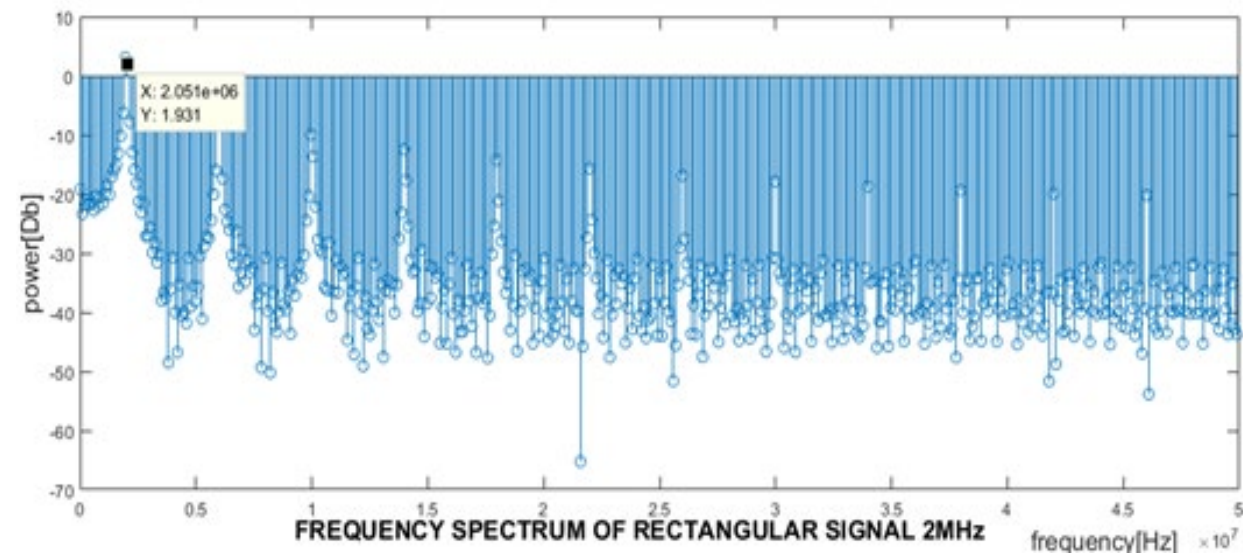

Fig. 4. Frequency domain 
- Amplitude modulated signal described in item 2.1

\section{Time domain in MATLAB}

figure (5); 응

plot $(t, y) \circ$ graph of the time domain of the signal ' $y$ '

The time domain of the amplitude modulated signal described in item 2.1 is shown on Fig. 5.

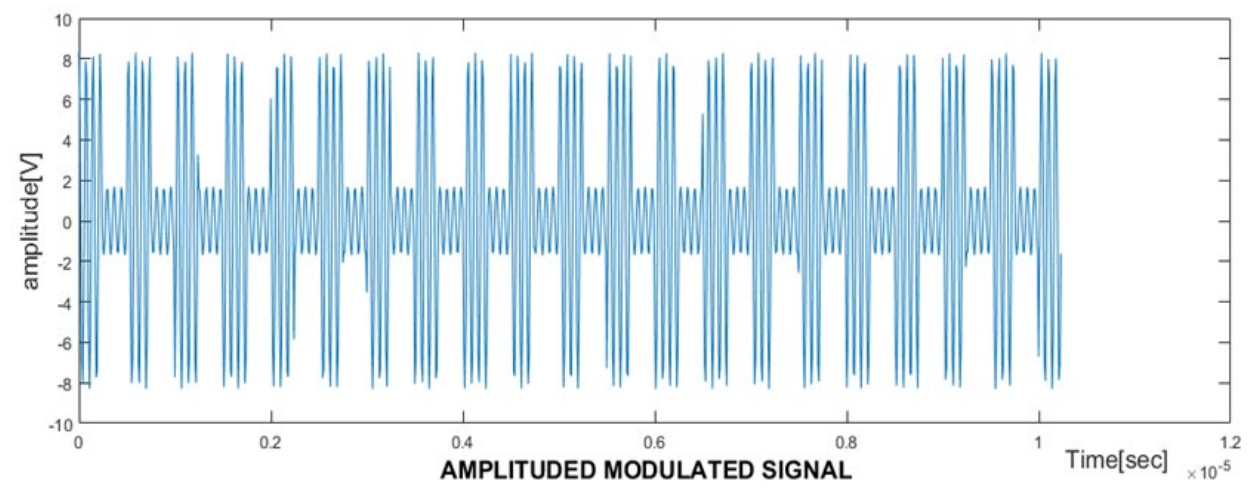

Fig. 5. Time domain

\section{Frequency domain in MATLAB}

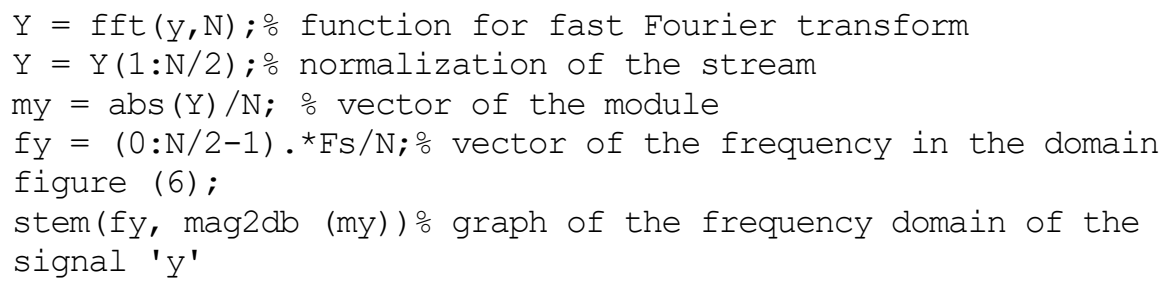

The frequency domain of the periodic rectangular signal described in item 2.1 is shown on Fig. 6 .

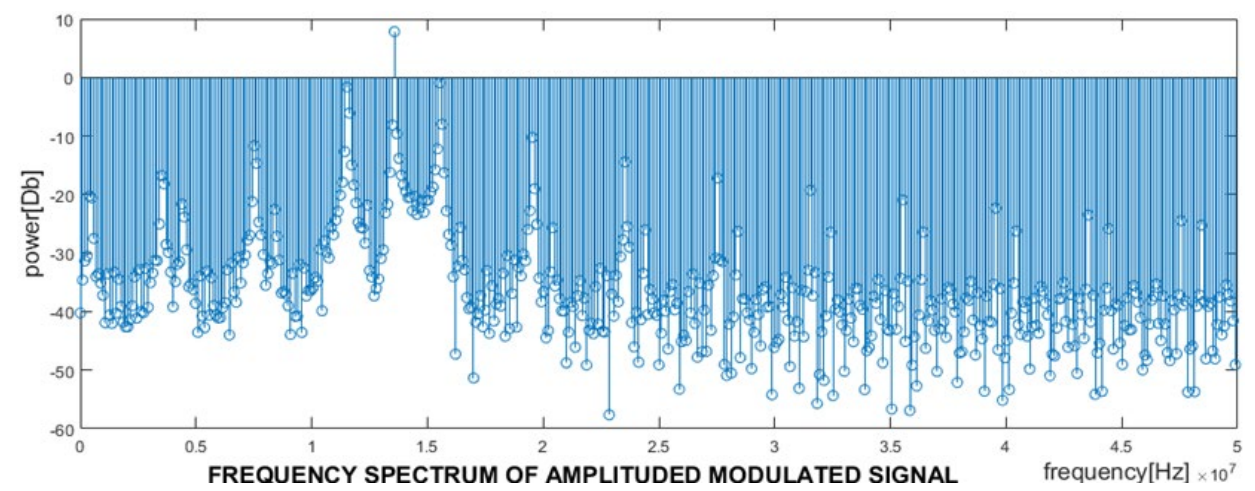

Fig. 6. Frequency domain 
- Amplitude modulated signal summed with a periodical sinusoidal signal described in item 2.1.

\section{Time domain in MATLAB}

figure (7); $\%$

plot $(t, y 1) \div$ graph of the time domain of the signal ' $y 1$ '

The time domain of the amplitude modulated signal summed with a periodical sinusoidal signal described in item 2.1 is shown on Fig. 7.

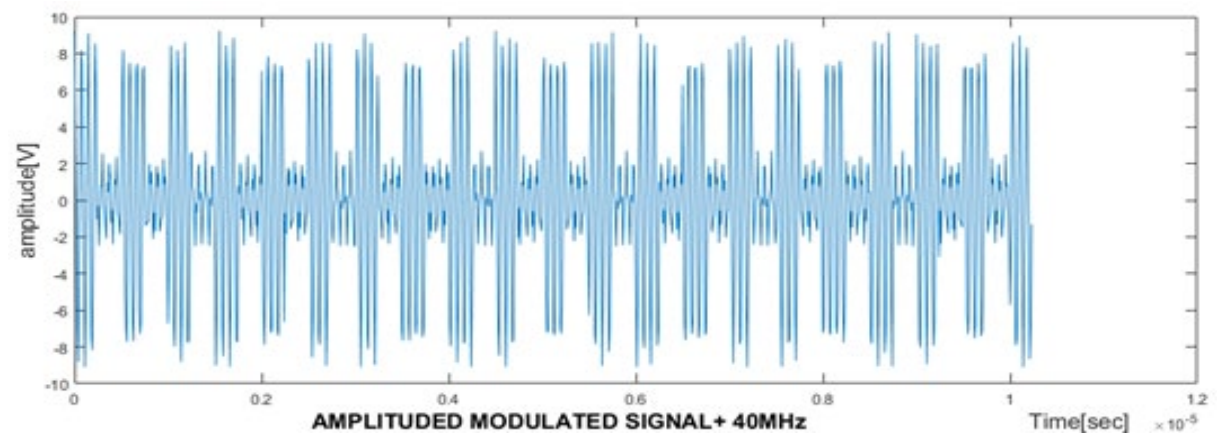

Fig. 7. Time domain

\section{Frequency domain in MATLAB}

$\mathrm{Y} 1=\mathrm{fft}(\mathrm{y} 1, \mathrm{~N}) ; \%$ function for fast Fourier transform

$\mathrm{Y} 1=\mathrm{Y} 1(1: \mathrm{N} / 2) ; \circ$ normalization of the stream

myl $=\operatorname{abs}(\mathrm{Y} 1) / \mathrm{N}$; $\frac{0}{\circ}$ vector of the module

fyl $=(0: \mathrm{N} / 2-1) .{ }^{\star} \mathrm{Fs} / \mathrm{N} ; \circ$ vector of the frequency in the domain

figure (8);

stem(fy1, mag2db (my1)) $\%$ graph of the frequency domain of the signal ' $y 1$ '

The frequency domain of the amplitude modulated signal summed with a periodical sinusoidal signal described in item 2.1 is shown on Fig. 8.

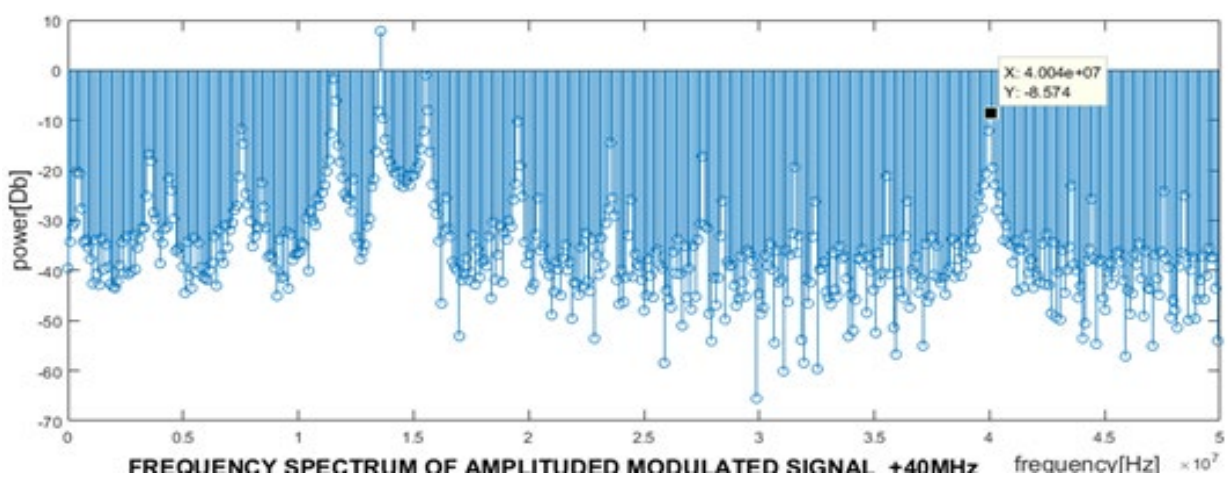

Fig. 8. Frequency domain 


\section{Filtering of Signals}

The useful signal is the one for developing an RFID system, [19, 20, 14]. The goal of developing a filter is partial of full removal of the excess part of the signal in the energy domain of the one. The frequency that will be used to achieve the communication for RFID systems is 13.56 [MHz]. This requirement limits the design space of a filter to use only passive components.

The design and development of a filter with an operational amplifier is not recommended because the channel capacity is at most $1[\mathrm{MHz}]$ as well as the price of a designed system increases then. More famous polynomial approximations are those of Butterworth, Chebyshev, Leander (Papoolis) and by Bessel (Thompson). The choice of an approximation depends on the required slope (with how many Decubels does the power of the signal drop) of the drop in Magnitude-Phase Representation (MPH).

\subsection{Development of a low-pass analog filter in the environment of MATLAB}

\section{Lo-pass filter Chebyshev in MATLAB:}

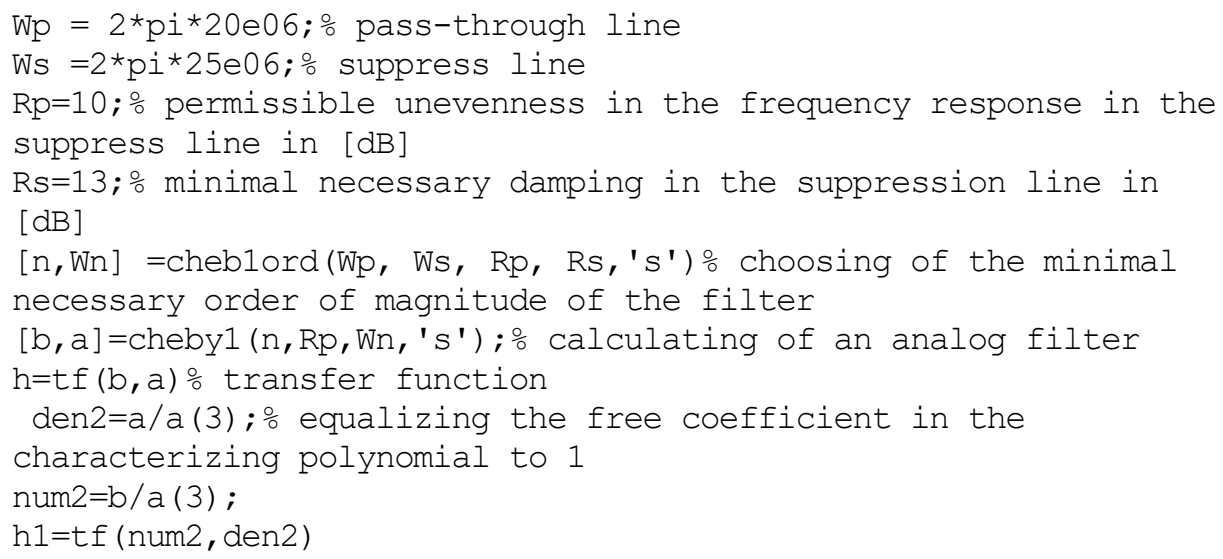

The transfer function of the model is:

$$
H(p)=\frac{0,3162}{1.202 e-16 p^{2}+3.512 e-09 p+1}
$$

\subsection{Mathematical model of a fluctuating unit from the second order of magnitude}

Transfer function of the oscillating unit is:

$$
W(P)=\frac{k}{T^{2} p^{2}-2 \xi T p+1}
$$


where $T$ is time constant of the linear system, $\xi$ is damping coefficient, $k-$ is amplification coefficient.

The mathematical model, [13] of a passive filter corresponds to a mathematical model of a fluctuating unit from the second order of magnitude.

Using relations (10) and (11), the following values of the variables are calculated: $T=1.0961 \mathrm{e}-08 ; \xi=0.1602 ; k=0.3162$.

\section{Check in MATLAB:}

pol=roots (a) $\%$ poles of the signal

nul=roots $(b) \div$ zeroes of the signal

$\mathrm{w} 1=\operatorname{sqrt}\left(\mathrm{real}(\mathrm{pol}(1))^{\wedge} 2+\operatorname{imag}(\mathrm{pol}(1))^{\wedge} 2\right) \div$ conjugate frequency in radian/seconds

$\mathrm{f}_{\mathrm{s}}=\mathrm{w} 1 /(2 * \mathrm{pi}) \div$ conjugate frequency in [Hz]

ksil=abs (real (pol(1)))/w1\% damping coefficient

$\mathrm{T} 1=1 / \mathrm{w} 1 \%$ time constant

The damping coefficient is $0<\xi<1$. That means that the previous function has damping fluctuations, which give us the opportunity to form a passive electrical circuit (Fig. 9).

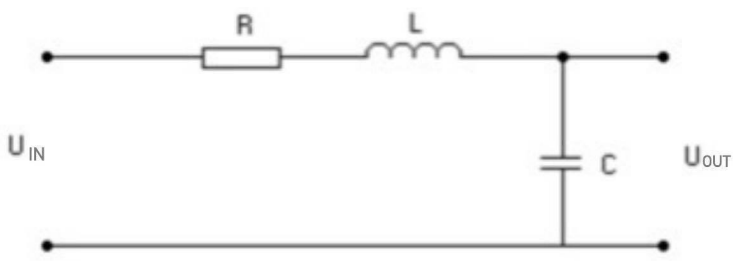

Fig. 9. Electrical circuit of a passive filter

The transfer function is then calculated after using the Kirchhoff's voltage law [21] like:

$$
W(p)=\frac{\frac{1}{\mathrm{pC}}}{\frac{1}{\mathrm{pC}}+\mathrm{pL}+\mathrm{R}}=\frac{1}{1+\mathrm{p}^{2} \mathrm{CL}+\mathrm{pCR}}
$$

From equations (10), (11), and (12) it is find the values of the parameters of the electrical circuit. The calculated values are shown in Table 1.

Table 1. Results for the values of the passive elements those are included in the electrical circuit of filter

\begin{tabular}{|c|c|c|c|c|c|c|c|c|}
\hline$n$ & $f_{\mathrm{s}}$ & $T$ & $\xi$ & $P o l$ & $k$ & $R$ & $L$ & $C$ \\
\hline- & $\mathrm{MHz}$ & $\mathrm{s}$ & - & - & - & $\Omega$ & $\mathrm{nH}$ & $\mathrm{pF}$ \\
\hline 2 & 14.520 & $1.0961 \mathrm{e}-08$ & 0.1602 & $\begin{array}{c}\text { Complex } \\
\text { conjugate } \\
-1.4613 \pm 9.0051 \mathrm{i}\end{array}$ & 0.3162 & 4 & 150 & 800 \\
\hline
\end{tabular}




\subsection{Reaction of a linear system to a random periodical signal in MATLAB}

- Function in MATLAB LSIM

$\mathrm{y}^{2}=\operatorname{ls} \operatorname{sim}(\mathrm{h} 1, \mathrm{y} 1, \mathrm{t})$; $\%$ Simulation of the time response of a dynamic system to a random input

The input signal for the filter is described in 2.2 and 3.1 [19].

- Graph representation in MATLAB

The frequency domain of the reaction of a linear system with a random periodical signal is shown on Fig. 10.

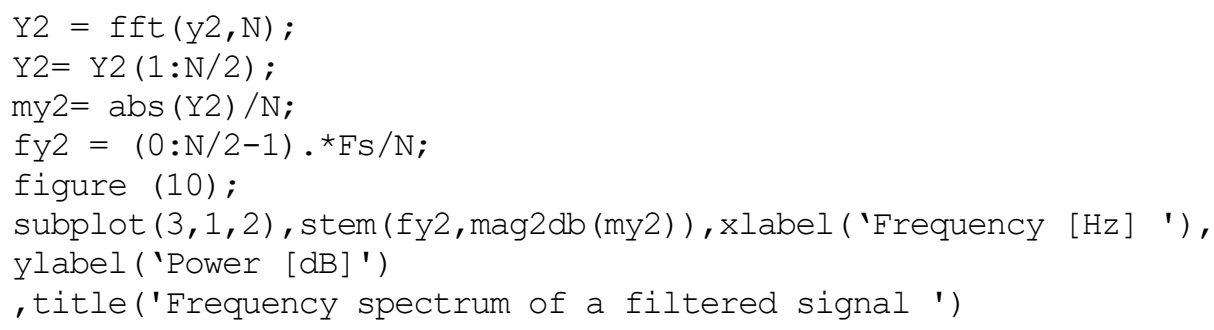

The graph representation of the reaction of the filter from 4.1. towards the signal modelled in 2.2 and 3.1 is shown on Fig. 10.

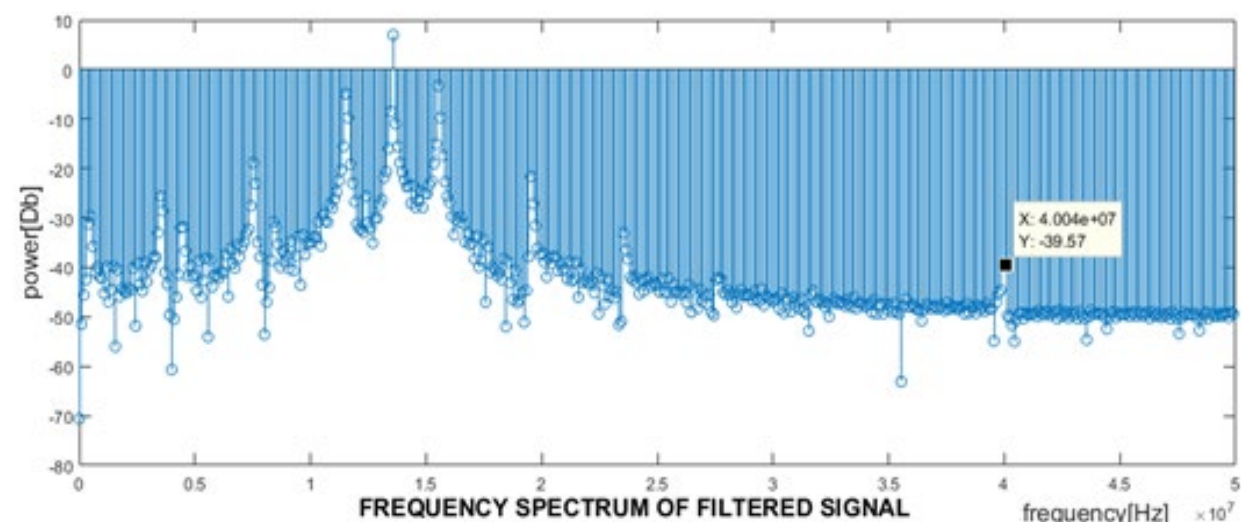

Fig. 10. The frequency spectrum

\section{Conclusion}

In this paper are described different stages for analysis when constructing the electrical circuit: electrical circuit analysis, frequency analysis, time analysis, modulation of signals and filtering of signals. The object of this paper are the technical methods for suppressing noise through filtering. Synthesized in such way, it gives clear insight into the work of passive filters visualized in the 
frequency and time domain simulated in the MATLAB environment. From the results it is clearly apparent that the power of the useless signal to us is 40 [MHz] and it has diminished by approximately $30[\mathrm{~dB}]$, which means that the passive filter that was used is effective in removing high-frequency signals from the spectrum of the useful signal.

The future investigations of described problems are related with providing a proper signals security control.

\section{Acknowledgments}

This work was supported by the Bulgarian Ministry of Education and Science under the National Research Program "Smart crop production” approved by Decision of the Ministry Council No 866/26.11.2020, (No D01-65/19.03.2021).

\section{References}

1. Borissova, D., Dimitrova, Z., Garvanova, M., Garvanov, I., Cvetkova, P., Dimitrov, V., Pandulis, A.: Two-stage decision-making approach to survey the excessive usage of smart technologies. Problems of Engineering Cybernetics and Robotics 73, 3-16 (2020), https://doi.org/10.7546/PECR.73.20.01.

2. Vodyaho, A., Yoshinov, R., Zhukova, N., Thaw, A.M., Ahmed Saddam A.: Fog oriented model for data collection in the networks of mobile devices. In: 2020 IEEE 10th International Conference on Intelligent Systems (IS), pp. 421-425 (2020), https://doi.org/10.1109/IS48319.2020.9200138.

3. Moysiadis, V., Sarigiannidis, P., Vitsas, V., Khelifi, A.: Smart farming in Europe. Computer Science Review 39, 100345 (2021), https://doi.org/10.1016/j.cosrev.2020.100345.

4. Trifonov, R., Tsochev, G, Yoshinov, R., Manolov, S., Pavlova, G.: Conceptual model for cyber intelligence network security system. International Journal of Computers 11, 85-92 (2017).

5. Kirchev, L., Blyantov, M., Georgiev, V., Boyanov, K.: A communication model supporting process migration in grid. In: Proc. EXPGRID workshop on largescale distributed systems of the 15th international symposium on HighPerformance Distributed Computing, Paris, France, pp. 31-39 (2006).

6. Tianxing, M., Yoshinov, R., Osipov, V., Zhukova, N., Schukina, M., Evnevich, E.: Problems of human secure interaction with the Internet space. Problems of Engineering Cybernetics and Robotics 75, 15-34 (2021), https://doi.org/10.7546/PECR.75.21.03.

7. Garvanova, M., Garvanov, I., Borissova D.: The influence of electromagnetic fields on human brain. In: Proc. of 21 st International Symposium on Electrical 
Apparatus \& Technologies (SIELA), pp. 1-4, (2020), https://doi.org/10.1109/SIELA49118.2020.9167099.

8. Behar, V., C. Kabakchiev, Doukovska, L.: Target trajectory detection in monopulse radar by Hough transform. Comptes rendus de l'Academie bulgare des Sciences 53(5), 45-48 (2000).

9. Doukovska, L.: Moving target Hough detector in pulse jamming. Cybernetics and Information Technologies 7(1), 67-76 (2007).

10. Doukovska, L.: Combined Doppler-Hough method for velocity estimation. In: Proc. of the International Radar Symposium - IRS'09, Hamburg, Germany, pp. 677-682 (2009).

11. Doukovska, L., Angelova, D.: Comparative analysis of two techniques for moving target velocity estimation. In: Proc. of the 7-th European Radar Conference - EuRAD'10, Paris, France, pp. 431-434 (2010).

12. Doukovska, L.: Track-before-detect procedure using Hough velocity estimation technique. Comptes rendus de l'Academie bulgare des Sciences 68(9), 11531160 (2015).

13. Doukovska, L.: Conventional Hough detector in presence of randomly arriving impulse interference. In: Proc. of the International Radar Symposium - IRS'15, Dresden, Germany, vol. 1, pp. 487-492 (2015).

14. Murata Manufacturing Co., Ltd, https://www.murata.com/en-eu.

15. Jose Maria Giron-Sierra. Digital Signal Processing with Matlab Examples. (2017).

16. Oppenheim, A. B., Wilsky, A. C., Young, J. T.: Signals \& Systems. Prentice Hall, (1996).

17. MATLAB - Control System Toolbox-User's Guide, http://www.mathworks.com.

18. MATLAB - Communications System Toolbox-User's Guide, http://www.mathworks.com.

19. MATLAB - Signal Processing Toolbox-User's Guide, http://www.mathworks.com.

20. MATLAB - Symbolic Math Toolbox-User's Guide, http://www.mathworks.com.

21. Nikolov, E.: Technical means for automation, Parts 1 and 2, TU-Sofia (2009). 